\title{
XLVIII. On electromagnetic waves, especially in relation to the vorticity of the impressed forces; and the forced vibrations of electromagnetic systems
}

\section{Oliver Heaviside}

To cite this article: Oliver Heaviside (1888) XLVIII. On electromagnetic waves, especially in relation to the vorticity of the impressed forces; and the forced vibrations of electromagnetic systems, Philosophical Magazine Series 5, 26:162, 434-449, DOI: $10.1080 / 14786448808628290$

To link to this article: http://dx.doi.org/10.1080/14786448808628290

央 Published online: 29 Apr 2009.

Submit your article to this journal $₫$

山l Article views: 6

Q View related articles $\longleftarrow$ 
434 Mr. O. Heaviside on Electromagnetic Waves, and the

Naphthaquinone :-

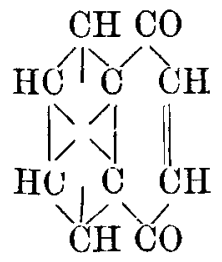

Fig. 13.

The oxidation not further affecting the second ring.

Tetrahydronaphthalene :-<smiles>CCC1(C)C2CCC3(C)CCCC231</smiles>

Fig. 14.

The addition not going further than to the extent of 4 univalent atoms.

I have adopted in this paper a method of treatment of the carbon atom in space somewhat different from the original conception of it as an atom situated at the centre and having its affinities directed towards the angles of a regular tetrahedron, in that $I$ have treated it as itself representable by a regular tetrahedron having its four affinities acting at the angles. It is, I think, a natural and perfectly legitimate method of treatment, and is in full accordance with Wislicenus' conception, when he states (Ber. xxi. p. 584), "Ich halte es nicht für unmöglich dass das Kohlenstoffatom ein Gebilde sei welche in seiner Gestalt einem regulären Tetraëder mehr oder weniger vielleicht recht sehr ähnelt."

XIVIII. On Electromagnetic Waves, especially in relation to the Vorticity of the Impressed Forces; and the Forced Vibrations of Electromagnetic S'ystems. By Oliver Heaviside.

[Continued from p. 382.]

Cylindrical Electromagnetic Waves.

49. T $\mathrm{N}$ concluding this paper I propose to give some cases of 1 cylindrical waves. They are selected with a view to the avoidance of mere mathematical developments and unin- 
telligible solutions, which may be multiplied to any extent; and for the illustration of peculiarities of a striking character. The case of vibratory impressed E.M.F. in a thin tube is very rich in this respect, as will be seen later. At present I may remark that the results of this paper have little application in telegraphy or telephony, when we are only concerned with long waves. Short waves are, or may be, now in question, demanding a somewhat different treatment*. We do, however, have very short waves in the discharge of condensers, and in vacuum-tube experiments, so that we are not so wholly removed from practice as at first appears. But independently of considerations of practical realization, I am strongly of opinion that the study of very unrealizable problems may be of use in forwarding the supply of one of the pressing wants of the present time or near future, a practicable æthermechanically, electromagnetically, and perhaps also gravitationally comprehensive.

50. Mathematical Preliminary.-On account of some peculiarities in Bessel's functions, which require us to change the form of our equations to suit circumstances, it is desirable to exhibit separately the purely mathematical part. This will also considerably shorten and clarify what follows it.

Let the axis of $z$ be the axis of symmetry, and let $r$ be the distance of any point from it. Either the lines of $\mathbf{E}$, electric force, or of $\mathrm{H}$, magnetic force, may be circular, centred on the axis. For definiteness, choose $\boldsymbol{H}$ here. Then the lines of $\mathbf{E}$ are either longitudinal, or parallel to the axis ; or there is, in addition, a radial component of $\mathbf{E}$, parallel to $r$. Thus the tensor $\mathrm{H}$ of $\mathrm{H}$, and the two components of $\mathrm{E}$, say $\mathrm{E}$ longitudinal and $\mathrm{F}$ radial, fully specify the field. Their connexions are these special forms of equations (2) and (3):-

* The waves here to be considered are essentially of the same nature as those considered by J. J. Thomson, "On Electrical Oscillations in a Cylindrical Conductor," Proc. Math. Soc. vol. xvii., and in Parts I. and II. of my paper "On the Self-Induction of Wires," Phil. Mugr. August and September 1886; viz. a mixture of the plane and cylindrical. But the peculiarities of the telegraphic problem nake it practically a case of plane waves as regards the dielectric, and cylindrical in the wires. The "resonance" effects described in my just-mentioned paper arise from the to-and-fro reflexion of the plane waves in the dielectric, moving parallel to the wire. This is also practically true in Prof. Lodge's recent experiments, discharging a Leyden jar into a miniature telegraph-circuit. On the other hand, most of such effects in the present paper depend upon the eylindrical waves in the dielectrie; and, in order to allow the dielectric fair play for their development, the contaminating influence of diffusion is done away with by using tubes only when there are cunductors. In Hertz's recent experiments the waves are of a very mixed character indeed. 


$$
\left.\begin{array}{c}
\frac{1}{r} \frac{d}{d r} r \mathrm{H}=(4 \pi k+c p) \mathrm{E},-\frac{d \mathrm{H}}{d z}=(4 \pi k+c p) \mathrm{F}, \\
\frac{d \mathrm{E}}{d r}-\frac{d \mathrm{~F}}{d z}=\mu p \mathrm{H},
\end{array}\right\}
$$

where (and always later) $p$ stands for $d / d t$. This is in space where neither the impressed electric nor the impressed magnetic force has curl, it being understood that $\mathbf{E}$ and $\mathbf{H}$ are the forces of the fluxes, so as to include impressed. From (302) we obtain

$$
\left.\begin{array}{l}
\frac{1}{r} \frac{d}{d r} r \frac{d \mathrm{E}}{d r}+\frac{d^{2} \mathrm{E}}{d z^{2}}=(4 \pi k+c p) \mu p \mathrm{E}, \\
\frac{d}{d r} \frac{1}{r} \frac{d}{d r} r \mathrm{H}+\frac{d^{2} \mathrm{H}}{d z^{2}}=(4 \pi k+c p) \mu p \mathrm{H},
\end{array}\right\}
$$

the characteristics of $\mathrm{E}$ and $\mathrm{H}$. Let now

$$
\left.q^{2}=-s^{2}=(4 \pi k+c p) \mu p-d^{2}\right) d z^{2} ; \quad . \quad .
$$

then the first of (303) becomes the equation of $J_{0}(s r)$ and its companion, whilst the second becomes that of $\mathrm{J}_{1}(s r)$, and its companion. Thus $\mathrm{E}$ is associated with $\mathrm{J}_{0}$ and $\mathrm{H}$ with $\mathrm{J}_{1}$, when $\mathbf{H}$ is circular; conversely when $\mathbf{E}$ is circular.

We have first Fourier's cylinder function

$$
\mathrm{J}_{\mathrm{or}}=\mathrm{J}_{0}(s r)=1-\frac{(s r)^{2}}{2^{2}}+\frac{(s r)^{4}}{2^{2} 4^{2}}-\ldots ; .
$$

and its companion, which call $\mathrm{G}_{0}$, is

$\mathrm{G}_{0 r}=\mathrm{G}_{0}(s r)=(2 / \pi)\left[\mathrm{J}_{0 r} \log s r+\mathrm{L}_{0 r}\right]$,

where

$$
\left.\mathrm{L}_{0 r}=\frac{(s r)^{2}}{2^{2}}-\left(1+\frac{1}{2}\right) \frac{(s r)^{4}}{2^{2} 4^{2}}+\left(1+\frac{1}{2}+\frac{1}{3}\right) \frac{(s r)^{6}}{2^{2} 4^{2} 6^{2}}-\ldots\right\}
$$

The coefficient $2 / \pi$ is introduced to simplify the solutions. The function $J_{1}(s r)$ or $J_{1}$ is the negative of the first derivative of $\mathrm{J}_{0 r}$ with respect to $s r$. Let $\mathrm{G}_{1}(s r)$ or $\mathrm{G}_{1 r}$ be the function similarly derived from $G_{0 r}$. The conjugate property, to be repeatedly used, is

$$
\left(\mathrm{J}_{0} \mathrm{G}_{1}-\mathrm{J}_{1} \mathrm{G}_{0}\right)_{r}=-2 / \pi s r . \quad . \quad .
$$

We have also Stokes's formula for $J_{o r}$, useful when $s r$ is real and not too small, viz.

$$
\mathrm{J}_{0 r}=(\pi s r)^{-\frac{1}{2}}[\mathrm{R}(\cos +\sin ) s r+\mathrm{S} i(\sin -\cos ) s r], .
$$

where $\mathbf{R}$ and $\mathrm{S} i$ are functions of $s r$ to be presently given. The corresponding formula for $\mathrm{G}_{0 r}$ is obtained by changing cos to $\sin$ and $\sin$ to $-\cos$ in $(308)$. 
Besides these two sets of solutions, we sometimes require to use a third set. A pair of solutions of the $J_{0}$ equation is

$$
\mathrm{U}=r^{-\frac{1}{2}} \epsilon^{q r}(\mathrm{R}+\mathrm{S}), \mathrm{W}=r^{-\frac{1}{4}} \epsilon^{-q r}(\mathrm{R}-\mathrm{S}), \text { ) }
$$

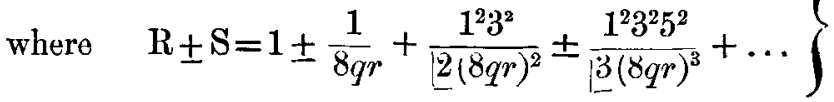

The last also defines the $R$ and $S i$ in (308). $R$ is real whether $q^{2}$ be + or - , whilst $\mathrm{S}$ is unreal when $q^{2}$ is - , or $\mathrm{S} i$ is then real, $s^{2}$ being + .

When $q r$ is a + numeric, the solution $U$ is meaningless, as its value is infinity. But in our investigations $q^{2}$ is a differential operator, so that the objection to $\mathrm{U}$ on that score is groundless. We shall use it to calculate the shape of an inward progressing wave, whilst $W$ goes to find an outward wave. The results are fully convergent within certain limits of $r$ and $t$. From this alone we see that a comprehensive theory of ordinary linear differential equations is sometimes impossible. They must be generalized into partial differential equations before they can be understood.

The conjugate property of $U$ and $W$ is

$$
\mathrm{UW}^{\prime}-\mathrm{U}^{\prime} \mathrm{W}=-2 q / r, \cdot \cdot \cdot
$$

if the ${ }^{\prime}=d / d r . \quad$ An important transformation sometimes required is

$$
\mathrm{J}_{0 r}-i \mathrm{G}_{0 r}=2 i \mathrm{~W}(2 \pi q)^{-\frac{\lambda}{2}} ; . . . .
$$

or, which means the same,

$$
\mathrm{W}=-\left(\frac{2 q}{\pi}\right)^{\frac{1}{2}}\left[\mathrm{~J}_{0 r} \log q r+\mathrm{L}_{0 r}\right] . \quad .
$$

When we have obtained the differential equation in any problem, the assumption $s^{2}=\mathrm{a}+$ constant converts it into the solution due to impressed force sinusoidal with respect to $t$ and $z$; this requires $d^{2} / d z^{2}=-m^{2}$, and $d^{2} / d t^{2}$ $=-n^{2}$, where $m$ and $n$ are positive constants, being $2 \pi$ times the wave-shortness along $z$ and $2 \pi$ times the frequency of vibration respectively.

After (309) we became less exclusively mathematical. To go further in this direction, and come to electromagnetic waves, observe that we need not concern ourselves at all with $F$ the radial component, in seeking for the proper differential equation connected with a surface of curl of impressed force ; it is $\mathrm{E}$ and $\mathrm{H}$ only that we need consider, as the boundary conditions concern them. The second of (302) derives $\mathrm{F}$ from $\mathrm{H}$. 
When $\mathrm{H}$ is circular, the operator $\mathrm{E} / \mathrm{H}$ is given by

$$
\frac{\mathrm{E}}{\mathrm{H}}=\frac{s}{4 \pi k+c p} \frac{\mathrm{J}_{0 r}-y \mathrm{G}_{0 r}}{\mathrm{~J}_{1 r}-y \mathrm{G}^{1 r}}, \quad . \quad . \quad .
$$

where $y$ is undetermined. When $\mathrm{E}$ is circular, the operator $\mathrm{E} / \mathrm{H}$ is given by

$$
\frac{\mathrm{E}}{\mathrm{H}}=\frac{s}{4 \pi k+c p} \frac{J_{1 r}-y \mathrm{G}_{1 r}}{J_{0 r}-y \mathrm{G}_{0 r}} . . . . .
$$

The use of these operators greatly facilitates and systematizes investigation. The meaning is that (313) or (314) is the characteristic equation connecting $\mathrm{E}$ and $\mathrm{H}$.

51. Longitudinal Impressed E.M.F. in a thin Conducting Tube.-Let an infinitely long thin conducting tube of radius $a$ have conductance $\mathrm{K}$ per unit of its surface to longitudinal current, and be bounded by a dielectric on both sides. Strictly speaking the tube should be infinitely thin, in order to obtain instantaneous magnetic penetration, and yet be of finite conductance without possessing infinite conductivity, because that would produce opacity. In this tube let impressed electric force, of intensity $e$ per unit length, act longitudinally, $e$ being any function of $t$ and $z$. We have to connect $e$ with $\mathrm{E}$ and $\mathrm{H}$ internally and externally.

The magnetic force being circular, (313) is the resistance operator required. Within the tube take $y=0$ if the axis is to be included ; else find $y$ by some internal boundary condition. Outside the tube take $y=i$ when the medium is homogeneous and boundless, because that is the only way to prevent waves from coming from infinity; else find $y$ by some outer boundary condition. There is no difficulty in forming the $y$ to suit any number of coaxial cylinders possessing different electrical constants, by the continuity of $E$ and $\mathrm{H}$ at each boundary, which equalizes the $\mathrm{E} / \mathrm{H}$ 's of its two sides, and so expresses the $y$ of one side in terms of that on the other; but this is useless for our purpose. For the present take $y=0$ inside, and leave it unstated outside.

At $r=a, \mathrm{E}_{a}$ has the same value on both sides of the tube, on account of its thinness. In the substance of the tube $e+\mathrm{E}_{a}$ is the force of the flux. On the other hand $\mathrm{H}$ is discontinuous at the tube, thus

$$
4 \pi \mathrm{K}(e+\mathrm{E})=\mathrm{H}_{\text {(out) }}-\mathrm{H}_{(\mathrm{in})}=\left(\frac{\mathrm{H}}{\overline{\mathrm{E}}} \text { (out) }-\frac{\mathrm{H}}{\overline{\mathrm{E}}}(\mathrm{in})\right) \mathrm{E}_{\boldsymbol{a}} .
$$

In this use (313), and the conjugate property (307), and we at once obtain

$$
e=\left[-1+\frac{4 \pi k+c p}{4 \pi \mathrm{K} s} \frac{2 y}{\pi s a} \frac{1}{J_{0 a}\left(J_{0 a}-y \mathrm{G}_{0 a}\right)}\right] \mathrm{E}_{a}, .
$$


from which all the rest follows. Merely remarking concerning $k$ that the realization of (316) when $k$ is finite requires the splitting up of the Bessel functions into real and imaginary parts, that the results are complex, and that there are no striking peculiarities readily deducible; let us take $k=0$ at once, and keep to non-conducting dielectrics. Then, from (316), follow the equations of $\mathrm{F}$ and $\mathrm{H}$, in and out; thus

$$
\begin{aligned}
& \mathrm{E}_{\text {(in) }} \text { or } \text { (out) }=\frac{\mathrm{J}_{0 r}\left(\mathrm{~J}_{0 a}-y \mathrm{G}_{0 a}\right) \text { or } \mathrm{J}_{0 a}\left(\mathrm{~J}_{0 r}-y \mathrm{G}_{0 r}\right)}{\frac{c p}{4 \pi \mathrm{K} s} \frac{2 y}{\pi s a}-\mathrm{J}_{0 a}\left(\mathrm{~J}_{0 a}-y \mathrm{G}_{0 a}\right)} e . \\
& \mathrm{H}_{\text {(in) }} \text { or }{ }_{\text {(out) }}=\frac{c p}{s} \frac{\mathrm{J}_{1 r}\left(\mathrm{~J}_{0 a}-y \mathrm{G}_{0 a}\right) \text { or } \mathrm{J}_{0 a}\left(\mathrm{~J}_{1 r}-y \mathrm{G}_{1 r}\right)}{\text { same denominator }}, .
\end{aligned}
$$

which we can now examine in detail.

52. Vanishing of Eixternal Field. $J_{0 a}=0$.- The very first thing to be observed is that $J_{0 a}=0$ makes $\mathrm{E}$ and $\mathrm{H}$ and therefore also $F$ vanish outside the tube, and that this property is independent of $y$, or of the nature of the external medium. We require the impressed force to be sinusoidal or simply periodic with respect to $z$ and $t$, thus

so that ultimately

$$
e=e_{0} \sin (m z+\alpha) \sin (n t+\beta), \quad . \quad .
$$

$$
s^{2}=n^{2} / v^{2}-m^{2} ; \quad \text {. . . . . . }
$$

and any one of the values of $s$ given by $\mathrm{J}_{0 a}=0$ causes the evanescence of the external field. The solutions just given reduce to

$$
\left.\begin{array}{l}
\mathrm{H}=-4 \pi \mathrm{K}\left(\mathrm{J}_{1 r} / \mathrm{J}_{1 a}\right) e \\
\mathrm{E}=(s / c n) 4 \pi \mathrm{K}\left(\mathrm{J}_{0 r} / \mathrm{J}_{1 a}\right) i e \\
\mathrm{~F}=-(c n)^{-1} 4 \pi \mathrm{K}\left(\mathrm{J}_{1 r} / \mathrm{J}_{1 a}\right) i(d e / d z)
\end{array}\right\} . .
$$

which are fully realized, becanse $i$ signifies $p / n$, or involves merely a time-differentiation performed on the $e$ of (319).

The electrification is solely upon the inner surface of the tube. In its substance $\mathrm{H}$ falls from $-4 \pi \mathrm{K} e$ inside to zero outside, and $\mathrm{E}_{a}$ being zero, the current in the tube is $\mathrm{K} e$ per unit surface.

The independence of $y$ raises suspicion at first that (321) may not represent the state which is tended to after $e$ is started. But since the resistance of the tube itself is sufficient to cause initial irregularities to subside to zero, even were there a perfectly reflecting barrier outside the tube to prevent dissipation of these irregularities in space, there seems no reason to doubt that (321) do represent the state asymprotically tended to. Changing the form of $y$ will only change 


\section{Mr. O. Heaviside on Electromagnetic Waves, and the}

the manner of the settling down. We may commence to change the nature of the medium immediately at the outer boundary of the tube. We cannot, however, have those abrupt assumptions of the steady or simply periodic state which characterize spherical waves, owing to the geometrical conditions of a cylinder.

53. Case of two Coaxial Tubes.-If there be a conducting tube anywhere outside the first tube, there is no current in it, except initially. From this we may conclude that if we transfer the impressed force to the outer tube, there will be no current in the inner. Thus, let there be an outer tube at $r=x$, of conductance $\mathrm{K}_{1}$ per unit area, containing the impressed force $e_{1}$. We have

$$
\mathrm{E}_{x}=\frac{4 \pi \mathrm{K}_{1} e_{1}}{\mathrm{Y}_{3}-\mathrm{Y}_{2}-4 \pi \mathrm{K}_{1}}, . . . . .
$$

where $Y_{3}$ and $Y_{2}$ are the $H / E$ operators just outside and inside the tube, whilst $\mathrm{E}_{x}$ is the $\mathrm{E}$ at $x$, on either side of the tube, resulting from $e_{1}$. Wo have

$$
\mathrm{Y}_{3}=\frac{c p}{s} \frac{\mathrm{J}_{1 x}-y_{1} \mathrm{G}_{1 x}}{\mathrm{~J}_{0 x}-y_{1} \mathrm{G}_{0 x}}, \quad \mathrm{Y}_{2}=\frac{c p}{s} \frac{\mathrm{J}_{1 x}-y \mathrm{G}_{1 x}}{J_{0 x}-y \mathrm{G}_{0 x}}, \quad .
$$

where $y_{1}$ is settled by some external and $y$ by some internal condition. In the present case the inner tube at $r=a$, if it contains no impressed force, produces the condition

$$
\mathrm{Y}_{2}-\mathrm{Y}_{1}=4 \pi \mathrm{K} \text { at } r=a, . . .
$$

where $Y_{1}$ is the internal $\mathrm{H} / \mathrm{E}$ operator. Or

giving

$$
4 \pi \mathrm{K}=\frac{c p}{s}\left(\frac{\mathrm{J}_{1 a}-y \mathrm{G}_{1 a}}{\mathrm{~J}_{0 a}-y \mathrm{G}_{0 a}}-\frac{\mathrm{J}_{1 a}}{\mathrm{~J}_{0 a}}\right)
$$

$$
y=\frac{4 \pi \mathrm{KJ}^{2}{ }_{0 a}}{\frac{2}{\pi s a} \frac{c p}{s}+4 \pi \mathrm{KJ}_{0 a} \mathrm{G}_{0 a}} . . . .
$$

Now, using (323) in (322) brings it to

$$
\mathrm{E}_{x}=\frac{\left(\mathrm{J}_{0 x}-y \mathrm{G}_{0 x}\right)\left(\mathrm{J}_{0 x}-y_{1} \mathrm{G}_{0 x}\right) 4 \pi \mathrm{K}_{1} e_{1}}{\frac{c p}{s}\left(y_{1}-y\right) \frac{2}{\pi s x}-4 \pi \mathrm{K}_{1}\left(\mathrm{~J}_{0 x}-y \mathrm{G}_{0 x}\right)\left(\mathrm{J}_{0 x}-y_{1} \mathrm{G}_{0 x}\right)},
$$

in which $y$ is given by (325), and from (326) the whole state due to $e_{1}$ follows, as modified by the inner tube.

Now $J_{0 a}=0$ makes $y=0$; this reduces (326) to 


$$
\mathrm{E}_{x}=\frac{J_{n x}\left(\mathrm{~J}_{0 x}-y_{1} \mathrm{G}_{0 x}\right) 4 \pi \mathrm{K}_{1} e_{1}}{\frac{c p}{s} y_{1} \frac{2}{\pi s x}-4 \pi \mathrm{K}_{1} \mathrm{~J}_{0 x}\left(\mathrm{~J}_{0 x}-y_{1} \mathrm{G}_{0 x}\right)} ; \quad .
$$

and, by comparison with (317) we see that it is now the same as if the inner tube were non existent. That is, when it is situated at a nodal surface of $\mathrm{E}$ due to impressed force in the outer tube, and there is therefore no current in it (except transversely, to which the dissipation of energy is infinitely small), its presence does nothing, or it is perfectly transparent.

It is clearly unnecessary that the external impressed force should be in a tube. Let it only be in tubular layers, without specification of actual distribution or of the nature of the medium, except that it is in layers so that $\varepsilon, k$, and $\mu$ are functions of $r$ only ; then if the axial portion be nonconducting dielectric, the $J_{0 r}$ function specifies $E$ and allows there to be nodal surfaces, for instance $J_{0 a}=0$, where a conducting tube may be placed without disturbing the field. Admitting this property $a b$ initio, we can conversely conclude that $e$ in the tube at $r=a$ will, when $J_{0 a}=0$, make every external cylindrical surface a nodal surface, and therefore produce no external disturbance at all.

54. Now go back to $\S 51$, equations (317) (318). There are no external nodal surfaces of $\mathrm{E}$ in general (exception later). We cannot therefore find a place to put a tube so as not to disturb the existing field due to $e$ in the tube at $r=a$. But we may now make use of a more general property. To illustrate simply, consider first the electromagnetic theory of induction between linear circuits. Let there be any number of circuits, all containing impressed forces, producing a determinate varying electromagnetic field. In this field put an additional circuit of infinite resistance. The E.M.F. in it, due to the other circuits, will cause no current in it of course, so that no change in the field takes place. Now, lastly, close the circuit or make its resistance finite, and simultaneously put in it impressed force which is at every moment the negative of the E.M.F. due to the other circuits. Since no current is produced there will still be no change, or everything will go on as if the additional circuit were nonexistent.

Applying this to our tubes, we may easily verify by the previous equations that when there are two coaxial tubes, both containing impressed forces, we can reduce the resultant electromagnetic field everywhere to that due to the impressed force in one tube, provided we suitably choose the impressed 
force in the second to be the negative of the electric force of field due to $e$ in the first tube when the second is nonexistent. That is, we virtually abolish the conductance of the second tube and make it perfectly transparent.

55. Perfectly Reflecting Barrier. Its efferts. Vanishing of Conduction Current.-To produce nodal surfaces of $\mathrm{E}$ outside the tube containing the vibrating impressed force, we require an external barrier, which shall prevent the passage of energy or its absorption, by wholly reflecting all disturbances which reach it. Thus, let there be a perfect conductor at $r=x$. This makes $\mathrm{E}=0$ there. This requires that the $y$ in (317), (318) shall have the value $\mathrm{J}_{0 x} / \mathrm{G}_{0 x}$, whereas without any bound to the dielectric it would be $i$. We can now choose $m$ and $n$ so as to make $\mathrm{J}_{0 x}=0$. This reduces those equations to

(in and out)

$$
\left.\begin{array}{l}
\mathrm{E}=-\frac{\mathrm{J}_{0 r}}{J_{0 a}} e ; \quad \mathrm{F}=-\frac{1}{s} \frac{\mathrm{J}_{1 r}}{s} e ; \\
\mathrm{H}=-\frac{1}{s} \frac{\mathrm{J}_{1 r}}{\mathrm{~J}_{0 a}} e p e .
\end{array}\right\} \cdot \cdot \cdot
$$

This solution is now the same inside and outside the tube containing the impressed force, and there is no current in the tube, that is, no longitudinal current.

To understand this case, take away the impressed force and the tube. Then (328) represents a conservative system in stationary vibration. Now, by the preceding, we may introduce the tube at a nodal surface of $\mathrm{E}$ without disturbing matters, provided there be no impressed force in the tube. But if we introduce the tube anywhere else, where $\mathbf{E}$ is not zero, we require, by the preceding, an impressed force which is at every moment the negative of the undisturbed force of the field, in order that no change shall occur. Now this is precisely what the solution (328) represents, $e$ in the tube being cancelled by the force of the field, so that there is no conduction-current. 'The remarkable thing is that it is the impressed force in the tube itself that sets up the vibrating field, and gradually ceases to work, so that in the end it and the tube may be removed without altering the field. That a perfect conductor as reflector is required is a detail of no moment in its theoretical aspect.

Shifting the tube, with a finite impressed force in it, towards a nodal suface of $E$, sends up the amplitude of the vibrations to any extent.

$56 . \mathrm{K}=0$ and $\mathrm{K}=\infty$. - If the tube have no conductance, $e$ produces no effect. This is because the two surfaces of 
curl of e are infinitely close together, and therefore cancel, not having any conductance between them to produce a discontinuity in the magnetic force.

But if the tube have infinite conductance, we produce complete independence between the internal and extermal fields, except in the quite unessential particnlar that the two surfaces of curl e are of opposite kind and time together. Equations $(317),(318)$ reduce to

$$
\mathrm{E}=-\frac{\mathrm{J}_{0 r}}{\mathrm{~J}_{1 a}^{-i}} e, \quad \mathrm{~F}=-\frac{1}{s} \frac{\mathrm{J}_{1 r}}{\mathrm{~J}_{0 a}} e, \quad \mathrm{H}=-\frac{1}{s} \frac{\mathrm{J}_{1 r}}{\mathrm{~J}_{0 a}} e p e .
$$

$$
\text { (out) }\left\{\begin{array}{c}
\mathrm{E}=-\frac{\mathrm{J}_{0 r}-y \mathrm{G}_{0 r}}{\mathrm{~J}_{0 a}-y \mathrm{G}_{0 a}, \quad \mathrm{~F}=\frac{1}{s} \frac{\mathrm{J}_{1 r}-y \mathrm{G}_{1 r}}{\mathrm{~J}_{0 a}-y \mathrm{G}_{0 a}},} \\
\mathrm{H}=-\frac{1}{s} \frac{\mathrm{J}_{1 r}-y \mathrm{G}_{1 r}}{\mathrm{~J}_{0 a}-y \mathrm{G}_{0 a}} c p e .
\end{array}\right\} \text {. }
$$

Observe that (329) is the same as (328). The external solution (330) requires $y$ to be stated. When $y=i$, for a boundless dielectric, the realization is immediate.

57. $s=0 . \quad V$ anishing of $\mathrm{E}$ all over, and of $\mathrm{F}$ and $\mathrm{H}$ also internally.-This is a singularity of quite a different kind. When $n=m v$, we make $s=0$. Of course there is just one solution with a given wave-length along $z$ : a great frequency with small wave-length, and conversely.

$\mathrm{E}$ vanishes all over, that is both inside and outside the tube containing $e$, provided $s / y$ is zero. The internal $H$ and therefore also $\mathrm{F}$ vanish. Thus within the tube is no disturbance, and outside, (317) (318) reduce to

$$
\mathrm{H}=\frac{a}{r} 4 \pi \mathrm{K} e, \quad \mathrm{~F}=\frac{1}{c n} \frac{a}{r} 4 \pi \mathrm{K} i \frac{d e}{d z}, . \quad . \quad .
$$

Observe that $\mathrm{H}$ and $\mathrm{F}$ do not fluctuate or alternate along $r$, but that $H$ has the same distribution (out from the tube) as if $e$ were steady and did not vary along $z$.

A special case is $m=0$. Then also $n=0$, or $e$ is steady and independent of $z, F$ vanishes, and the first of (331) expresses the steady state.

Without this restriction, the current in the tube is $\mathrm{K} e$ per unit surface, owing to the vanishing of the opposing longitudinal $\mathrm{E}$ of the field. This property was, by inadvertence, attributed by me in a former paper* to a wire instead of a

\footnotetext{
* “On Resistance and Conductance Operators," Phil. Mag. Dec. 1887, p. $492, \operatorname{Ex} . j$.
} 


\section{$444 \mathrm{Mr}$. O. Heaviside on Electromagnetic Waves, and the}

tube. The wave-length must be great in order to render it applicable to a wire, because instantaneous penetration is assumed.

I mentioned that $s / y$ must vanish. This occurs when $y=i$, or the external dielectric is boundless. But it also occurs when $\mathrm{E}=0$ at $r=x$, produced by a perfectly conductive screen. This is plainly allowable because it does not interfere with the $\mathrm{E}=0$ all over property. What the screen does is simply to terminate the field abruptly. Of course it is electrified.

58. $s=0$ and $\mathrm{H}_{x}=0$. - But with other boundary conditions, we do not bave the solutions (331). Thus, let $\mathrm{H}_{x}=0$, instead of $\mathbf{E}_{x}=0$. This makes $y=J_{1 x} / \mathbf{G}_{1 x}$ in (317), (318). There are at least two ways (theoretical) of producing this boundary condition. First, there may be at $r=x$ a screen made of a perfect magnetic conductor $(g=\infty)$. Or, secondly, the whole medium beyond $r=x$ may be infinitely elastive and resistive $(c=0, k=0)$ to an infinite distance.

Now choose $s=0$ in addition and reduce (317), (318). The results are

$$
\left.\begin{array}{rl}
\mathrm{E} & =-\frac{e}{1+\frac{1}{2} x^{2} c p / 4 \pi \mathrm{K} a} ; \quad \mathrm{F}=-\frac{1}{c p} \frac{d \mathrm{H}}{d z} \\
\text { (in) or (out) } \mathrm{H} & =-\frac{c p e}{1+\frac{1}{2} x^{2} c p / 4 \pi \mathrm{K} a}\left(\frac{r}{2} \text { or } \frac{r}{2}-\frac{x^{2}}{2 r}\right),
\end{array}\right\}
$$

which are at once realized by removing $p$ from the denominator to the numerator.

Although $\mathbf{E}$ is not now zero, it is independent of $r$, only varying with $t$ and $z$.

When $s^{2}$ is negative, or $n<m / v$, the solutions (317), (318) require transforming in part because some of the Bessel functions are unreal. Use (312), because $q$ is now real. There are no alternations in $\mathrm{E}$ or $\mathrm{H}$ along $r$. They only commence when $n>n v$.

59. Separate actions of the two surfaces of curl e.-Since all the fluxes depend solely upon the curl of $e$ and not upon its distribution, and there are two surfaces of curl $e$ in the tabe problem, their actions, which are independent, may be separately calculated. The inner surface may arise from $e$ in the - direction in the inner dielectric, or by the same in the + direction in the tube and beyond it. The outer may be due to $e$ in the - direction beyond the tube, or in the + direction in the tube and inner dielectric.

We shall easily find that the inner surface of curl of e, say of surface density $f_{1}$, produces 
$\left.\begin{array}{l}\text { (in) } \mathrm{E}=\mathrm{J}_{0 r} \frac{\left(\mathrm{J}_{1 a}-y \mathrm{G}_{1 a}\right)-\left(\mathrm{J}_{0 a}-y \mathrm{G}_{0 a}\right) 4 \pi \mathrm{K} s / c p}{2 y / \pi s a-\mathrm{J}_{0 a}\left(\mathrm{~J}_{0 a}-y \mathrm{G}_{0 a}\right) 4 \pi \mathrm{K} s / c p} f_{1} \\ \text { (out) } \mathrm{E}= \\ \frac{\mathrm{J}_{1 a}\left(\mathrm{~J}_{0 r}-y \mathrm{G}_{0 r}\right)}{\text { same denominator }} f_{1}\end{array}\right\}$.

from which $\mathrm{H}$ may be got by the $\mathrm{E} / \mathrm{H}$ operator.

The external sheet, say $f_{2}$, produces

$$
\left.\begin{array}{l}
\text { (in) } \mathrm{E}=\frac{\mathrm{J}_{0 r}\left(\mathrm{~J}_{1 a}-y \mathrm{G}_{1 a}\right)}{\cdot \cdot \cdot \cdot} f_{2}, \\
\text { (out) } \mathrm{E}=\left(\mathrm{J}_{0 r}-y \mathrm{G}_{0 r}\right) \frac{\mathrm{J}_{1 a}+\mathrm{J}_{0 a} 4 \pi \mathrm{K} s / c p}{\cdot \cdot \cdot \cdot \cdot} f_{s}
\end{array}\right\} .
$$

where the unwritten denominators are as in the first of (333). Observe that when $\mathbf{J}_{1 a}=0, f_{1}$ produces no external field (in tube or beyond it). It is then only $f_{2}$ that operates in the tube and beyond.

Now take $f_{2}=e$ and $f_{1}=-e$ in (333) and (334) and add the results. We then obtain (317), (318); and it is now $\mathrm{J}_{0 a}=0$ that makes the external field vanish, instead of $J_{1 a}=0$ when $f_{1}$ alone is operative.

Having treated this problem of a tube in some detail, the other examples may be very briefly considered, although they too admit of numerous singularities.

60. Circular Impressed. Force in Conducting-tube.-The tube being as before, let the impressed force e (per unit length) act circularly in it instead of longitudinally, and let e be a function of $t$ only, so that we have an inner and an outer cylindrical surface of longitudinally directed curl of e. $\mathbf{H}$ is evidently longitudinal and $\mathbf{E}$ circular, so that we now require to use the (314) operator.

At the tube $\mathrm{E}_{a}$ is continuous, this being the tensor of the force of the flux on either side, and $H$ is discontinuous thus,

or

$$
\mathrm{H}_{\text {(in) }}-\mathrm{H}_{\text {(out) }}=4 \pi \mathrm{K}\left(e+\mathrm{E}_{a}\right) \text {, }
$$

$$
e=-\left\{1+\frac{1}{4 \pi \mathrm{K}}\left(\frac{\mathrm{H}}{\overline{\mathrm{E}}} \text { (out) }-\frac{\mathrm{H}}{\overline{\mathrm{E}}}(\text { in })\right)\right\} \mathrm{E}_{a} . .
$$

Substituting the (314) operator, with $y=0$ inside, and $y$ undetermined outside, and using the conjugate property (307), we obtain

$$
\begin{aligned}
& \mathrm{H}_{\text {(in) }} \text { or (out) }=-i \frac{\left(\mathrm{J}_{1 a}-y \mathrm{G}_{1 a}\right) \mathrm{J}_{0 r} \text { or } \mathrm{J}_{1 a}\left(\mathrm{~J}_{0 r}-y \mathrm{G}_{0 r}\right)}{\mu v \mathrm{~J}_{1 a}\left(\mathrm{~J}_{1 a}-y \mathrm{G}_{1 a}\right)+\frac{y}{4 \pi \mathrm{K}} \frac{2 v}{\pi a p}} e_{,}(336) \\
& \mathrm{E}_{\text {(in) }} \text { or } \text { (out) }=-\mu v \frac{\left(\mathrm{J}_{1 a}-y \mathrm{G}_{1 a}\right) \mathrm{J}_{1 r} \text { or } \mathrm{J}_{1 a}\left(\mathrm{~J}_{1 r}-y \mathrm{G}_{1 r}\right)}{\text { same denominator }} e .(337)
\end{aligned}
$$

Phil. Mag. S. 5. Vol. 26. No. 162. Nov. 1888. 
When $e$ is simply periodic, $J_{1 a}=0$ makes the external $\mathrm{E}$ and $H$ vanish independent of the nature of $y$. The complete solution is then

$$
\mathrm{H}_{(\mathrm{in})}=4 \pi \mathrm{K} \frac{\mathrm{J}_{0 \mathrm{r}}}{\mathrm{J}_{0 a}} e, \quad \mathrm{E}_{(\mathrm{in})}=-4 \pi \mathrm{K} \mu v \frac{\mathrm{J}_{1 r}}{\mathrm{~J}_{0 a}} i e . .
$$

The conduction-current in the tube is $\mathrm{K} e$ per unit area of surface.

To make the conduction-current vanish by balancing the impressed force against the electric force of the field that it sets up, put an infinitely conducting sereen at $r=x$ outside the tube and choose the frequency to make $J_{1 x}=0$, since we now have $y=\mathrm{J}_{1 x} / \mathrm{G}_{1: z^{*}}$. We shall then have the same solution inside and outside, viz.

$$
\mathrm{H}=-\frac{1}{\mu v} \frac{\mathrm{J}_{0 r}}{\mathrm{~J}_{1 a}} i e, \mathrm{E}=-\frac{\mathrm{J}_{1 r}}{\mathrm{~J}_{1 a}} e ; . . .
$$

so that at the tube itself $\mathrm{E}=-e$. This case may be interpreted as in $\$ 55$, the tube being at a nodal surface of $\mathrm{E}$.

A special case of (338) is when $n=0$, or e is steady. Then there is merely the longitudinal $\mathrm{H}$ inside the tube, given by

$$
\mathrm{H}=4 \pi \mathrm{K} e \text {. }
$$

61. Cylinder of longitudinal curl of $\mathrm{e}$ in a Dielectric.In a nonconductive dielectric let the impressed electric force be such that its curl is confined to a cylinder of radius $a$, in which it is uniformly distributed, and is longitudinal. Let $f$ be the tensor of curl $e$, and let it be a function of $t$ only. Since $\mathbf{E}$ is circular and $\mathbf{H}$ longitudinal, we have (314) as operator, in which $k$ is to be zero. This is outside the cylinder. Inside, on the other hand, on account of the existence of curl $e$, the equation corresponding to (314) is

$$
\frac{\mathrm{E}}{\mathrm{H}-f / \mu p}=\frac{s}{c p} \frac{\mathrm{J}_{1 r}}{J_{0 r}} \cdot . \quad \cdot \quad \cdot \quad \cdot .
$$

At the boundary $r=a$ both $\mathrm{E}$ and $\mathrm{H}$ are continuous; so, by taking $r=a$ in (340) and in the corresponding (314) with $k=0$, and eliminating $\mathrm{E}_{a}$ or $\mathrm{H}_{a}$ between them, we obtain the equation of the other. We obtain

$$
\text { (out) }\left\{\begin{array}{l}
\mathrm{E}=\frac{1}{2} \pi a y^{-1} \mathrm{~J}_{1 a}\left(\mathrm{~J}_{1 r}-y \mathrm{G}_{1 r}\right) f, \\
\mathrm{H}=\frac{1}{2} \pi a y^{-1} \mathrm{~J}_{1 a}\left(\mathrm{~J}_{0 r}-y \mathrm{G}_{0 r}\right)(\mu v)^{-1} i f,
\end{array}\right\} \ldots
$$

in which $y$, as usual, is to be fixed by an external boundary condition, or, if the medium be boundless, $y=i$.

We see at once that $J_{1 a}=0$, with $f$ simply periodic, makes the external fluxes vanish. We should not now say that it 
makes the external field vanish, though the statement is true as regards $\mathrm{H}$, because the electric force of the field does not vanish; it cancels the impressed force, so that there is no flux. This property is apparently independent of $y$. But, since there is no resistance concerned, except such as may be expressed in $y$, it is clear that (341) sinusoidally realized cannot represent the state which is tended to after starting $f$, unless there be either no barrier, so that initial disturbances can escape, or else there be resistance somewhere, to be embodied in $y$, so that they can be absorbed, though only through an infinite series of passages between the boundary and the axis of the initial wave and its consequences.

Thus, with a conservative barrier producing $\mathrm{E}=0$ at $r=x$, and $y=J_{1 x} / G_{1 x}$, there is no escape for the initial effects, which remain in the form of free vibrations, whilst only the forced vibrations are got by taking $s^{2}=+$ constant in (341). The other part of the solution must be separately calculated. If $\mathrm{J}_{1 x}=0, \mathrm{E}$ and $\mathrm{H}$ run up infinitely. If $\mathrm{J}_{1 u}=0$ also, the result is ambiguous.

With no barrier at all, or $y=i$, we have

$$
\text { out }\left\{\begin{array}{l}
\mathrm{E}=-(2 a)^{-1} \mathrm{~J}_{1 a}\left(\mathrm{G}_{1 r}+i \mathrm{~J}_{1 r}\right) f_{0}, \\
\mathrm{H}=(2 a \mu v)^{-1} \mathrm{~J}_{1 a}\left(\mathrm{~J}_{0 r}-i \mathrm{G}_{0 r}\right) f_{0},
\end{array}\right\}
$$

which are fully realized. Here $f_{0}=f \pi a^{2}$, which may be called the strength of the filament. We may most simply take the impressed force to be circular, its intensity varying as $r$ within and inversely as $r$ outside the cylinder. Then $f=2 e / a$, if $e_{a}$ is the intensity at $r=a$.

When $n r / v$ is large, (342) becomes, by (308),

$$
\text { (out) } \mathrm{E}=\mu v \cdot \mathrm{H}=\frac{f_{0} n}{4 v}\left(\frac{2 v}{\pi n r}\right)^{\frac{1}{2}} \sin \left(n t-\frac{n r}{v}+\frac{\pi}{4}\right) .
$$

approximately. $2 \pi r$ should be a large multiple, and $2 \pi a$ a small fraction of the wave-length along $r$.

62. Filament of curl e. Calculation of Wave.-In the last let $f_{0}$ be constant whilst $a$ is made infinitely small. It is then a mere filament of curl of $e$ at the axis that is in operation. We now have, by the second of (342), with $J_{1 a}=\frac{1}{2} n a / v$,

$$
\mathrm{H}=-(c p / 4)\left(i \mathrm{~J}_{0 r}+\mathrm{G}_{0 r}\right) f_{0}, \text {. . . . }
$$

which may be regarded as the simply periodic solution or as the differential equation of $\mathrm{H}$. In the latter case, put in terms of $W$ by (311), then

$$
\begin{gathered}
\mathrm{H}=(2 \mu v)^{-1}(g / 2 \pi)^{\frac{1}{2}} \mathrm{~W} f_{0} ; . . . \\
2 \mathrm{H} 2
\end{gathered}
$$


448 Mr. O. Heaviside on Electromagnetic Waves, and the or, expanding by (309),

$$
\mathrm{H}=\frac{1}{2 \mu v} \frac{1}{(2 \pi r)^{\frac{1}{2}}} \epsilon^{-q r}\left(1-\frac{1}{8 q r}+\frac{1^{2} 3^{2}}{\left[2(8 q r)^{2}\right.}-\ldots\right) q^{\frac{1}{2}} f_{0}, .
$$

in which $f_{0}$ may be any function of the time. Let it be zero before and constant after $t=0$. Then, first,

$$
q^{\frac{1}{2}} f_{0}=f_{0}(\pi v t)^{-\frac{1}{2}} \text {. . . . . . }
$$

Next effect the integrations of this function indicated by the inverse powers of $q$ or $p / v$, thus

$$
\begin{gathered}
f_{0}\left(1-\frac{1}{8 q r}+\ldots\right)(\pi v t)^{-\frac{1}{3}}=\left(1-\frac{1}{2}\left(\frac{v t}{2 r}\right)+\frac{1.3}{2^{2}[2}\left(\frac{v t}{2 r}\right)^{2}-\ldots\right)(\pi v t)^{-\frac{1}{2}} \\
=(1+v t / 2 r)^{-\frac{1}{2}}(\pi v t)^{-\frac{1}{2}}=(2 r / \pi)^{\frac{1}{2}}[v t(v t+2 r)]^{-\frac{1}{2}} . .(348)
\end{gathered}
$$

Lastly, operating on this by $\epsilon^{-q r}$ turns $v t$ to $v t-r$, and brings (346) to

$$
\mathrm{H}=\left(f_{0} / 2 \pi \mu v\right)\left(v^{2} t^{2}-r^{2}\right)^{-\frac{1}{2}}, \quad . \quad .
$$

which is ridiculously simple. Let $\mathrm{Z}$ be the time-integral of $\mathrm{H}$, then

$$
\mathrm{Z}=\frac{c f_{0}}{2 \pi} \log \left[\frac{v t}{r}+\left(\frac{v^{2} t^{2}}{r^{2}}-1\right)^{\frac{1}{2}}\right], \quad . \quad .
$$

from which we may derive $\mathrm{E}$; thus

$$
\operatorname{curl} \mathrm{Z}=c \mathrm{E}, \quad \text { or } \mathrm{E}=-\frac{1}{c} \frac{d \mathrm{Z}}{d r}=\frac{v t f_{0}}{2 \pi r\left(v^{2} t^{2}-r^{2}\right)^{\frac{1}{2}}} .
$$

The other vector-potential $\mathbf{A}$, such that $\mathbf{E}=-p \mathbf{A}$ is obviously

$$
\mathrm{A}=-\frac{1}{2 \pi v}\left(\frac{v^{2} t^{2}}{r^{2}}-1\right) . \quad . \quad . \quad .
$$

All these formulæ of course only commence when $v t$ reaches $r$. The infinite values of $E$ and $H$ at the wave-front arise from the infinite concentration of the curl of $e$ at the axis.

Notice that

$$
\mathrm{E}=\mathrm{H} t / r c \text {. . . . . . . }
$$

everywhere. It follows from this connexion between $\mathrm{E}$ and $\mathrm{H}$ (or from their full expressions) that

$$
c \mathrm{E}^{2}-\mu \mathrm{H}^{2}=c e^{2}=c\left(f_{0} / 2 \pi r\right)^{2} ; \quad . \quad .
$$

where $e$ denotes the intensity of impressed force at distance $r$, when it is of the simplest type, above described. That is, the 
excess of the electric over the magnetic energy at any point is independent of the time. Both decrease at an equal rate; the magnetic energy to zero, the electric energy to that of the final steady displacement $c e / 4 \pi$.

The above $\mathrm{E}$ and $\mathrm{H}$ solutions are fundamental, because all electromagnetic disturbances due to impressed force depend solely upon, and come from, the lines of curl of the impressed force. From them, by integration, we can find the disturbances due to any collection of rectilinear filaments of $f$. Thus, to find the $\mathrm{H}$ due to a plane sheet of parallel uniformly distributed filaments, of surface-density $f$, we have, by (349), at distance $a$ from the plane, on either side,

$$
\mathrm{H}=\int \frac{f d y}{2 \pi \mu v\left(v^{2} t^{2}-a^{2}-y^{2}\right)^{\frac{3}{2}}}=\frac{f}{2 \pi \mu v}\left[\sin ^{-1} \frac{y}{\left(v^{2} t^{2}-a^{2}\right)^{\frac{3}{2}}}\right],
$$

where the limits are $\pm\left(v^{2} t^{2}-a^{2}\right)^{\frac{1}{2}}$. Therefore

$$
\mathrm{H}=f / 2 \mu v
$$

after the time $t=a / v$; before then, $\mathrm{H}$ is zero.

Similarly, a cylindrical sheet of Jongitudinal f produces

$$
\mathrm{H}=\frac{f a}{2 \pi \mu v} \int \frac{d \theta}{\left(v^{2} t^{2}-b^{2}\right)^{\frac{3}{3}}} ;
$$

where $b$ is the distance of the point where $\mathrm{H}$ is reckoned from the element $a d \theta$ of the circular section of the sheet, $a$ being its radius. The limits have to be so chosen as to include all elements of $f$ which have had time to produce any effect at the point in question. When the point is external and $v t$ exceeds $a+r$ the limits are complete, viz. to include the whole circle. The result is then, at distance $r$ from the axis of the cylinder,

$$
\begin{array}{r}
\mathrm{H}=\frac{f a / \mu v}{\left(v^{2} t^{2}-a^{2}-r^{2}\right)^{\frac{1}{2}}}\left[1+\frac{1.3}{2^{2} \mid \frac{2}{2}} \frac{x}{2}+\frac{1.3 .5 .7}{2^{4} \mid \frac{1}{2}} \frac{x^{2}}{2^{3}} \frac{4.3}{1.2}\right. \\
\left.+\frac{1.3 .5 .7 .9 .11}{2^{6} \mid 6} \frac{x^{3}}{2^{5}} \frac{6.5 .4}{1.2 .3}+\ldots\right]
\end{array}
$$

where

$$
x=(2 a r)^{\frac{1}{2}}\left(v^{2} t^{2}-a^{2}-r^{2}\right)^{-\frac{1}{3}} .
$$

This formula begins to operate when $x=1$, or $v t=a+r$. As time goes on, $x$ falls to zero, leaving only the first term.

[To be continued.] 